\title{
Duloxetine in the treatment of generalized anxiety disorder
}

This article was published in the following Dove Press journal:

International Journal of General Medicine

18 August 2009

Number of times this article has been viewed

\author{
Alan Wright \\ Chad VanDenBerg \\ Center for Clinical Research, \\ Mercer University, Atlanta, \\ GA, USA
}

Correpondence: Chad VanDenBerg Director, Center for Clinical Research, Clinical Associate Professor, Mercer University, College of Pharmacy and Health Sciences, 300I Mercer University Drive, Atlanta, GA 3034I, USA Email vandenberg_c@mercer.edu

\begin{abstract}
Duloxetine is a serotonin-norepinephrine reuptake inhibitor (SNRI) which is FDA approved for the treatment of generalized anxiety disorder (GAD) in doses of $30 \mathrm{mg}$ to $120 \mathrm{mg}$ daily. Duloxetine has been shown to significantly improve symptoms of GAD as measured through the Hamilton Anxiety Rating Scale (HAMA), the Clinical Global Impressions Scale (CGI-I), and other various outcome measures in several placebo-controlled, randomized, double blind, multi-center studies. Symptom improvement began within the first few weeks, and continued for the duration of the studies. In addition, duloxetine has also been shown to improve outcomes in elderly patients with GAD, and in GAD patients with clinically significant pain symptoms. Duloxetine was noninferior compared with venlafaxine XR. Duloxetine was found to have a good tolerability profile which was predictable and similar to another SNRI, venlafaxine. Adverse events (AEs) such as nausea, constipation, dry mouth, and insomnia were mild and transient, and occurred at relatively low rates. It was found to have a low frequency of drug interactions. In conclusion, duloxetine, a selective inhibitor for the serotonin and norepinephrine transporters, is efficacious in the treatment of GAD, and has a predictable tolerability profile, with AEs generally being mild to moderate.
\end{abstract}

Keywords: duloxetine, generalized anxiety disorder, anxiety, GAD

\section{Introduction}

Generalized anxiety disorder (GAD) is a serious and potentially debilitating psychiatric disorder characterized by generalized and persistent excessive anxiety, accompanied by other psychological or somatic symptoms (eg, palpitations, sweating, sleep disturbances). ${ }^{1,2}$ Symptoms for GAD diagnosis, which must be present for at least 6 months (according to DSM-IV and ICD-10 criteria) can be found in Table 1.

GAD is a common disorder, with a lifetime and 12-month prevalence in the US of $4.1 \%$ and $2.1 \%$, respectively. ${ }^{3}$ In Europe, the lifetime prevalence was found to be similar at $4.3 \%$ to $5.9 \%{ }^{2}$ GAD often coexists with other psychiatric disorders, such as major depressive disorder (MDD), panic disorder (PD), or alcohol and substance abuse. ${ }^{1}$ The most common of these psychiatric comorbidities is MDD which is observed in almost two thirds of patients. ${ }^{4}$ As well as psychiatric comorbidities, GAD is also comorbid with a number of medical conditions including cardiovascular disease, stroke, peptic ulcer disease, irritable bowel syndrome, pain, and headache. ${ }^{1}$ The high prevalence of GAD and its comorbid conditions results in a substantial financial burden as well as a personal one. In fact, some estimates put the US economic cost of anxiety disorders, inclusive of GAD, at US\$42 to US\$63 billion per year. ${ }^{5,6}$ A recent retrospective review of healthcare claims in patients 
Table I Symptoms of generalized anxiety disorder ${ }^{1}$

\begin{tabular}{ll}
\hline Psychological symptoms & Physical symptoms \\
\hline Excessive anxiety & Fatigue \\
Inability to control worries & Restlessness \\
Difficulty concentrating & Muscle tension \\
Feeling "on edge" & Nausea and diarrhea \\
& Palpitations \\
& Sweating \\
\hline
\end{tabular}

with GAD found an average annual cost of US\$7,451 per patient, and that $77 \%$ of these patients had either comorbid pain or depression. ${ }^{7}$ The disease is almost twice as common in women than men, generally appears in childhood or late adolescence, and has a median duration of 15.6 years; ${ }^{8}$ however it can appear later in life, and is often precipitated by a life-changing event. ${ }^{8}$

It is hypothesized that GAD is the result of neurobiological dysfunction of the serotonergic, noradrenergic, or GABAergic systems, ${ }^{2}$ and medications that have an effect on these systems are able to reduce GAD symptoms. ${ }^{9}$ The use of buspirone, selective serotonin (5-HT) reuptake inhibitor (SSRI) antidepressants and/or benzodiazepines are commonly prescribed as first-line agents in treating GAD, followed by serotonin/norepinephrine reuptake inhibitor (SNRI) antidepressants, mirtazapine, or a monoamine oxidase inhibitor (MAOI). If unsuccessful with these medications, tricyclic antidepressants, anticonvulsants, and antipsychotics may be used. , $^{4,8,10,11}$ Psychotherapy, as monotherapy or combined with pharmacotherapy, has also been studied to treat GAD.$^{12}$ Psychotherapeutic approaches include cognitive behavioral therapy, psychodynamic therapy, and supportive therapy. ${ }^{12}$

Currently, there are two SNRIs approved by the FDA for the treatment of GAD - venlafaxine and, more recently, duloxetine. This article will review the efficacy of the SNRI duloxetine in the treatment of GAD.

\section{Duloxetine}

\section{Pharmacodynamics}

Duloxetine is a SNRI that is approved by the FDA for the treatment of MDD, neuropathic pain, fibromyalgia, and GAD. ${ }^{13}$ Its mechanism of action is based upon reuptake inhibition of 5-HT and norepinephrine (NE) in the CNS. ${ }^{14}$ A number of in vitro experiments show that duloxetine has good affinity for 5-HT and NE transporters, and is able to decrease 5-HT and NE uptake, ${ }^{15-17}$ Other preclinical studies demonstrate the low affinity that duloxetine has for other transporters (DA, GABA) ${ }^{18}$ and for other neuronal binding sites (monoamine receptor subtypes and ion channels). ${ }^{15}$ In addition to in vitro studies, various in vivo techniques have been used to demonstrate duloxetine's inhibitory capabilities at 5-HT and NE transporters. ${ }^{15,16}$

There are also a number of clinical studies which demonstrate the ability of duloxetine to inhibit reuptake of NE and 5-HT. ${ }^{14}$ Reuptake inhibition of 5-HT can be shown by the use of platelet 5-HT levels. Platelets have a similar mechanism of 5-HT reuptake to that found in the CNS, and therefore 5-HT levels in the blood depend on the extent of reuptake inhibition in platelets. Turcotte et al and Chalon et al have demonstrated this relationship by showing an increase in blood 5-HT levels using duloxetine doses of $20 \mathrm{mg}$ daily to $120 \mathrm{mg}$ daily. ${ }^{19,20}$ Since there is no direct measure of NE reuptake inhibition, of NE activity is assessed by measuring the amount of NE and NE metabolites in the urine, cardiovascular effects NE reuptake inhibition, and by reduction of pain symptoms and adverse drug effects from NE reuptake inhibition. ${ }^{14}$ Each of these methods has been used to successfully demonstrate the ability of duloxetine to inhibit NE reuptake. ${ }^{14}$

\section{Pharmacokinetics}

After a single $20.2 \mathrm{mg}$ dose of oral duloxetine, the mean time to peak plasma concentration $\left(\mathrm{C}_{\max }\right)$ is 6 hours. ${ }^{21}$ During a dose escalation study, 8 subjects received duloxetine $20 \mathrm{mg}$ twice daily, $30 \mathrm{mg}$ twice daily then $40 \mathrm{mg}$ twice daily. Steady state plasma concentrations were reached after 5 days and duloxetine has a mean half-life of 12.5 hours $^{22}$ (with a range of 8 to 17 hours); ${ }^{13}$ thus duloxetine is a suitable candidate for once daily dosing. In the same study, duloxetine was shown to follow first-order absorption and elimination kinetics, and have a mean oral clearance of $114 \mathrm{~L} /$ hour, and mean apparent volume of distribution of $1943 \mathrm{~L} .{ }^{21}$ Absorption of duloxetine is unaffected by food, ${ }^{13}$ which makes dosing convenient and aids medication adherence.

Duloxetine is greater than $90 \%$ plasma protein bound, so caution should be exercised when administered with other highly protein bound drugs such as warfarin or phenytoin. ${ }^{13}$ It is extensively metabolized by the liver via CYP1A2 and CYP2D $6{ }^{13}$ resulting in various metabolites, the most predominant of these being the glucuronide conjugate 4-hydroxy duloxetine. ${ }^{21}$ Its metabolism by the cytochrome P450 system leads to the potential of certain drug interactions, as discussed in the next section. None of the duloxetine metabolites appear to be pharmacologically active. ${ }^{13}$ Excretion occurs $72 \%$ via the kidneys and $19 \%$ via feces. ${ }^{21}$ 


\section{Drug-drug interactions}

Because of its metabolism by $1 \mathrm{~A} 2$ and $2 \mathrm{D} 6$, there is a potential for drug interactions to take place between duloxetine and inhibitors, inducers, or substrates of these enzymes. When duloxetine $60 \mathrm{mg}$ was co-administered with fluvoxamine $100 \mathrm{mg}$ (a $1 \mathrm{~A} 2$ inhibitor) as a single dose, the result was an approximately 6-fold increase in duloxetine AUC, 2.5-fold increase in $\mathrm{C}_{\max }$, and a 3 -fold increase in half-life. ${ }^{13}$ In another study, duloxetine $40 \mathrm{mg}$ daily at steady state conditions was co-administered with paroxetine $20 \mathrm{mg}$ (a potent 2D6 inhibitor). Pharmacokinetic analysis showed a 1.6-fold increase in both duloxetine AUC and $\mathrm{C}_{\max }{ }^{23}$ Duloxetine has been reported to have a small inhibitory effect on CYP1A2, but no effect on $2 \mathrm{C} 9,2 \mathrm{C} 19$ or $3 \mathrm{~A} 4 .{ }^{13,24}$ It has, however, been shown to have a significant inhibitory effect on CYP2D6. Thirteen subjects each received desipramine $50 \mathrm{mg}$ (a substrate for CYP2D6) after 21 days of duloxetine therapy. It was found that desipramine $\mathrm{C}_{\max }$ had increased 1.7-fold, and AUC had increased 2.9-fold. ${ }^{23}$ Another potential drug interaction involves the use of anticoagulants (owing to the small $1 \mathrm{~A} 2$ inhibition). ${ }^{25}$ Pharmacodynamic drug interactions may occur with MAOIs, and other serotonergic drugs, resulting in an increased risk of serotonin syndrome. An example of this involved an interaction between duloxetine and linezolid. Linezolid has been shown to be a nonselective inhibitor of monoamine oxidase. ${ }^{26}$ It is recommended that patients be monitored closely if taking duloxetine concomitantly with any of these drug classes. ${ }^{13}$

\section{Efficacy in GAD}

A Medline search was made using the keywords duloxetine, Cymbalta $^{\circledR}$, generalized/generalised anxiety disorder, efficacy, and controlled trial. Five controlled trials for the use of duloxetine in GAD were found. The following section will summarize these trials.

Koponen et al carried out a multicenter, randomized, double blind, fixed-dose, placebo-controlled trial. ${ }^{27}$ Study sites were located in the US, Europe, and South Africa. The study was a 9-week treatment phase followed by a 2 -week discontinuation phase. All subjects participated in a pretreatment screening phase and washout period, and a 1-week single-blind placebo lead in phase. Subjects were diagnosed with GAD by a psychiatrist via the use of the Mini-International Neuropsychiatric Interview (MINI) and according to DSM-IV criteria. Subjects were excluded from the trial if the patient suffered from significant comorbidities or other mental disorders such as a recent diagnosis of MDD, substance abuse, post-traumatic stress disorder (PTSD), panic disorder, eating disorder, bipolar, obsessive compulsive disorder, or psychosis. Subjects were randomly assigned one of two active treatment groups: $60 \mathrm{mg}$ duloxetine daily, or $120 \mathrm{mg}$ duloxetine daily, along with a placebo group. The mean total Hamilton Rating Scale for Anxiety (HAM-A) scores at baseline were approximately 25 in all 3 groups, indicating moderately severe GAD.

The primary efficacy outcome measure was defined as a $>50 \%$ reduction in total HAM-A score from baseline, and sustained improvement rates were defined as a $>30 \%$ reduction in total HAM-A score from baseline to the last visit. Remission was defined as a $<7$ HAMA total score at endpoint. Secondary outcome measures were scores on the HAM-A psychic factor, the HAM-A somatic factor, the patient reported Hospital Anxiety and Depression Scale (HADS), the Clinical Global ImpressionsImprovement scale (CGI-I), the Patient Global ImpressionsImprovement scale (PGI-I), and the Sheehan Disability Scale (used to rate social, family, and work impairment).

Both treatment arms reported a significantly greater decrease in total HAM-A score as compared to placebo. The mean change from baseline was -12.8 in the $60 \mathrm{mg}$ group, -12.5 in the $120 \mathrm{mg}$ group, and -8.38 in the placebo group ( $P<0.001$ for both groups vs placebo). (Standard deviations were not reported). This corresponded to a mean decrease in total HAM-A score of $49 \%$ in duloxetine treated patients. A response was achieved by $58 \%$ of the duloxetine $60 \mathrm{mg}$ group, $56 \%$ of the duloxetine $120 \mathrm{mg}$ group, and $31 \%$ of the placebo group $(P<0.001$ both groups vs placebo), and remission was achieved by $31 \%, 38 \%$, and $19 \%$ of the duloxetine $60 \mathrm{mg}, 120 \mathrm{mg}$, and placebo groups, respectively $(P<0.01$ for duloxetine $60 \mathrm{mg}$ vs placebo; $P<0.001$ for duloxetine $120 \mathrm{mg}$ vs placebo). The duloxetine groups also had significantly greater sustained improvement rates as compared with the placebo group $-64 \%$ and $67 \%$ for the $60 \mathrm{mg}$ and $120 \mathrm{mg}$ groups respectively, vs $43 \%$ for placebo $(P<0.001$ both groups vs placebo). Secondary outcome measures also showed a greater improvement in the duloxetine groups over the placebo group (Table 2).

A similar study was completed by Rynn et $\mathrm{al}^{28}$ and with the same primary and secondary endpoints as Koponen et al. ${ }^{27}$ However, this study was conducted at sites exclusively in the US and consisted of a 10 week-long therapy phase rather than 9 weeks. Also, this study involved a titrated duloxetine dose as opposed to a fixed dose. All subjects participated in a pre-treatment screening phase and washout period, and a 1-week, single-blind placebo lead in phase. Subjects 


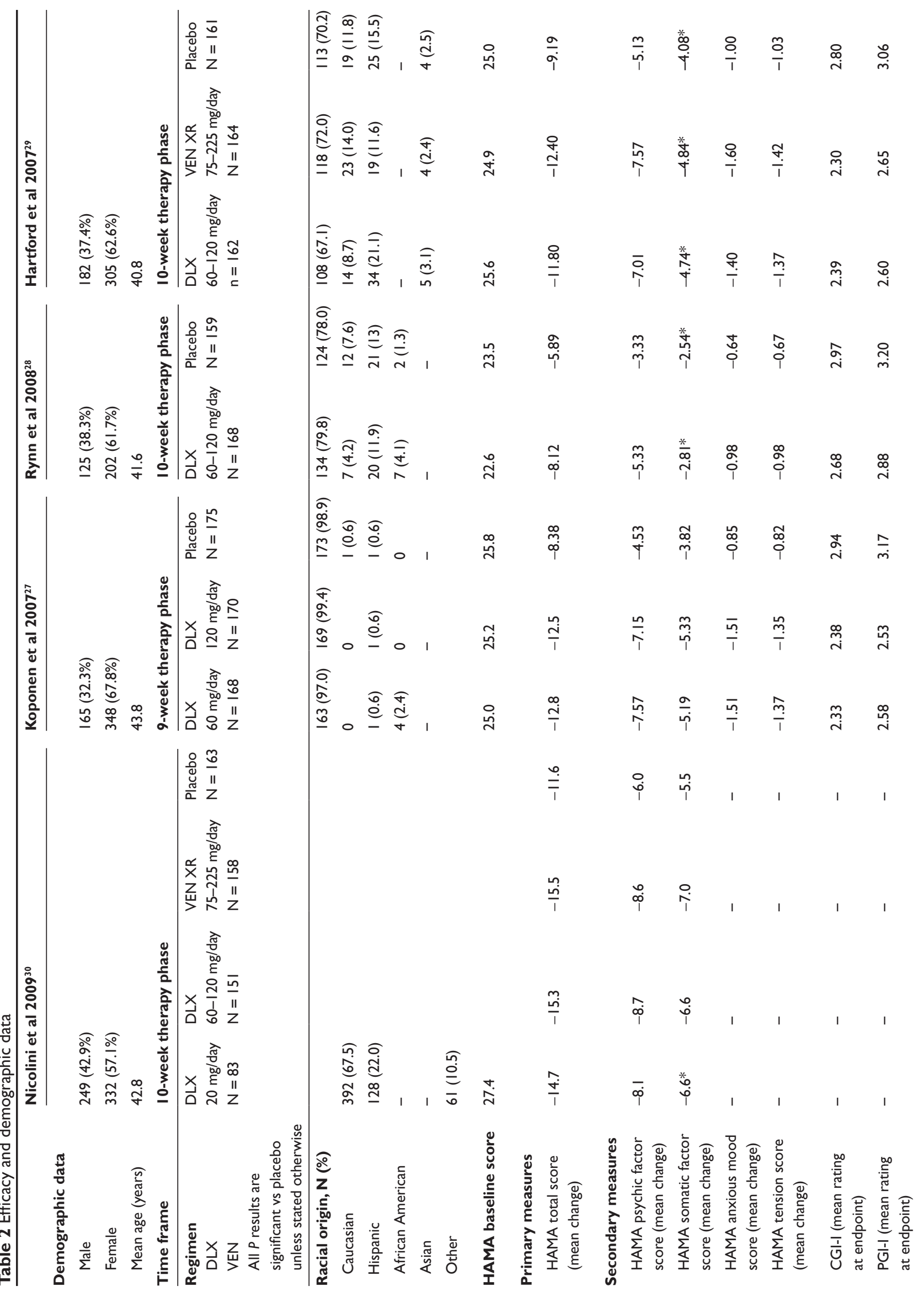




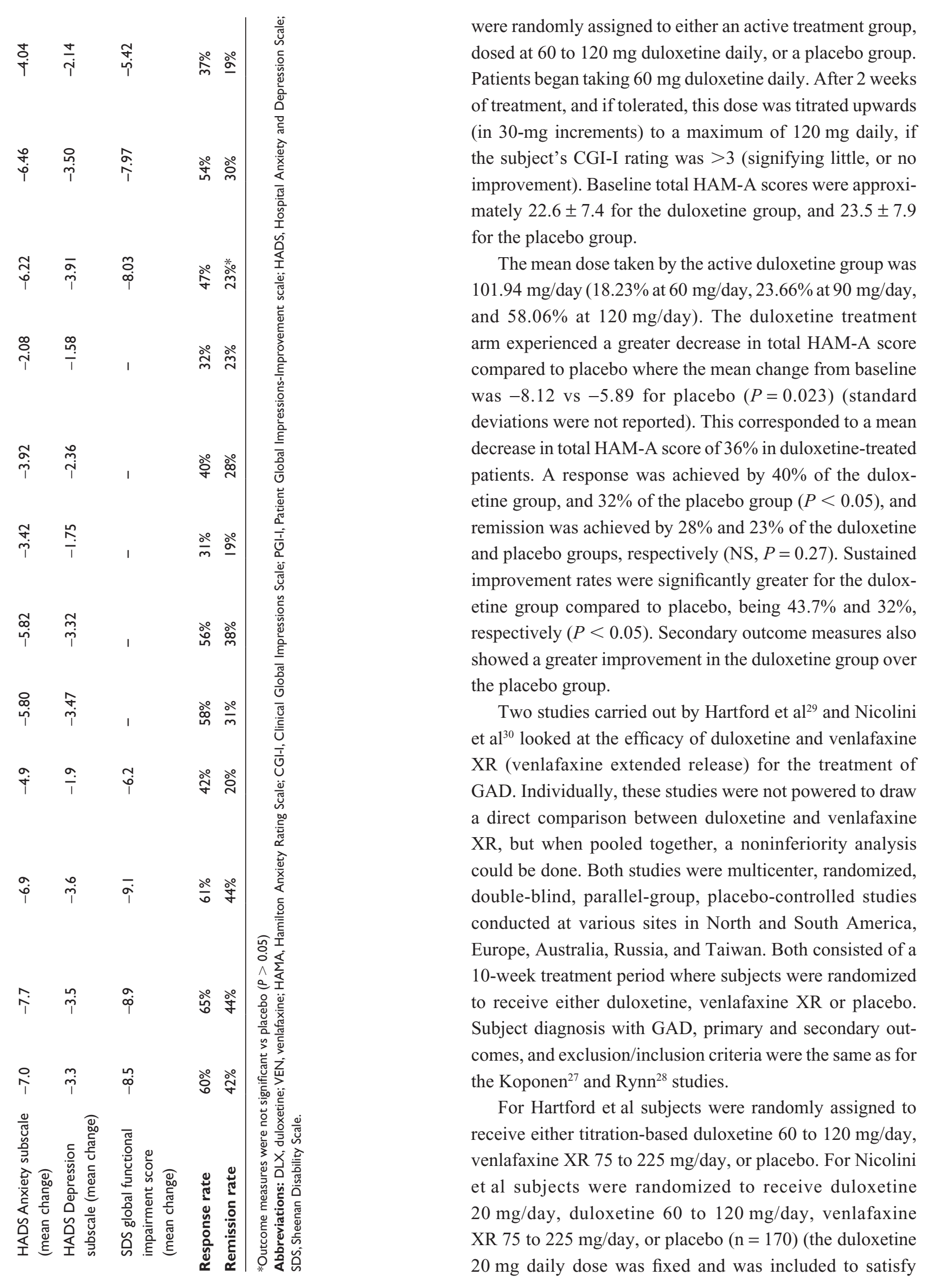


European regulatory requirements). For both studies, the duloxetine group daily dose was titrated in 30-mg increments to a maximum of $120 \mathrm{mg}$ daily, if tolerated. The venlafaxine group's doses were titrated from $75 \mathrm{mg}$ daily up to a maximum of $225 \mathrm{mg}$ daily, if tolerated. Doses were increased if there was no improvement in CGI-I score (ie, $\geq 3$ ).

At endpoint, the mean duloxetine doses being taken were $107.73 \mathrm{mg} /$ day for Hartford et al and $90 \mathrm{mg} /$ day for Nicolini et al. The duloxetine treatment arms experienced a greater decrease in total HAM-A score compared to placebo. The mean changes from baseline in the duloxetine 60 to $120 \mathrm{mg}$ groups were $-11.8 \pm 0.69$ (SE) for the Hartford et al study, and $-15.3 \pm 0.7(\mathrm{SE})$ for the Nicolini et al study, which corresponds to mean decreases in HAM-A of $46 \%$ and $55 \%$, respectively. The duloxetine $20 \mathrm{mg}$ group from the Nicolini et al study saw a HAM-A decrease of $14.7 \pm 1.0$ (SE) corresponding to a mean decrease of $45 \%$. All changes were statistically significant vs placebo. Efficacy and demographic data for the 4 studies discussed previously is summarized in Table 2.

In addition to the 4 acute clinical trials, a relapse prevention study of duloxetine in GAD was conducted. ${ }^{31}$ The study determined the relapse rate of GAD after discontinuation of duloxetine, and was a randomized, double blind, placebo-controlled trial, conducted at sites throughout the US and Europe. It consisted of a 26-week open label phase where 887 patients were enrolled to take duloxetine 60 to $120 \mathrm{mg}$ daily. This was done in a flexible dosing manner, whereby doses were increased from $60 \mathrm{mg}$ to $120 \mathrm{mg}$ daily if patients were non responsive (defined as a CGI-I $\leq 3$ ) and if medication could be tolerated. If subjects were responsive to their medication (defined as a $\geq 50 \%$ reduction in HAMA total score from baseline) they were randomized into the double-blind, placebo-controlled continuation phase of the study. Subjects who were randomized to receive placebo had their duloxetine dose tapered over a 2 -week period. The primary efficacy measure for this trial was time to relapse, with relapse defined by a $\geq 2$ point increase on the CGI-S scale and a score of $\geq 4$. Secondary measures were similar to the previous studies, and included HAM-A total score, HADS, and SDS. Out of the 887 subjects in the open label phase, 429 were randomized to take part in the continuation phase. Reasons for subject discontinuation included inadequate response during open label phase (9.7\%), adverse events (AEs) (13.6\%) and patient decision (12\%). Subjects remaining were assigned to either duloxetine (continued at the same dose as during the open-label phase) or placebo for 26 weeks.
Results showed that duloxetine was superior to placebo in preventing relapse of GAD over the 26-week period. Relapse criteria was met by $13.7 \%$ of the duloxetine group compared to $41.8 \%$ of the placebo group ( $P \leq 0.001$ ), and the mean change in HAM-A score from baseline was $7.5 \pm 0.6$ (SE) for the placebo group vs $1.6 \pm 0.6$ (SE) for the active group $(P \leq 0.001)$. For the patients who did relapse, those treated with placebo relapsed at an earlier time period than those treated with duloxetine $(P \leq 0.001)$.

\section{Special patient populations}

Using pooled data from previous studies the use of duloxetine was analyzed in specific patient populations. An analysis of duloxetine efficacy in treating GAD patients with clinically significant pain symptoms was conducted by Russell et $\mathrm{al}^{32}$ Data was pooled from the studies completed by Koponen et $\mathrm{al}^{27}$ and Rynn et $\mathrm{al}^{28}$ In both studies, pain was assessed using visual analogue scales (VAS) and statistical analysis was conducted in patients with a VAS score $>30$. This score was considered to be the point at which patients with little or no bodily pain was distinguishable from those with notable pain. Based upon these criteria, $44 \%$ of patients were identified in the intent to treat (ITT) group. Results of the analysis showed a significant decrease in overall VAS score for the duloxetine treated patients compared to placebo (mean change from baseline $48.7 \%$ vs $31.3 \%$; $P<0.001)$. There was also a direct correlation between reduction in VAS score and improvement in CGI-I scores. Patients with a final CGI-I score of 1 (max. improvement) had a mean reduction of $77.4 \%$ in total VAS score. Likewise, this was shown in the relationship between VAS score and PGI-I score, with patients having a final PGI-I score of 1 (maximum improvement) having a mean reduction in VAS score of $76.4 \%$. Given that GAD patients with pain have more severe symptoms, this analysis shows that duloxetine can deliver significant reductions in pain in patients with anxiety.

Another meta-analysis study assessed duloxetine's use in elderly patients with GAD. Clearly, pharmacotherapy in this population carries with it treatment concerns including polypharmacy, changes in drug metabolism, and other geriatric conditions such as Alzheimers disease. Davidson et $\mathrm{al}^{33}$ pooled data from four studies that were discussed previously. ${ }^{27-30}$ Out of the 1491 patients, data were taken from patients over 65 years of age, where 45 patients in the duloxetine group and 28 in the placebo group were found (73 patients in total equaling $4.9 \%$ of sample). All patient data were used to evaluate safety, and patients who completed 
at least one post baseline measurement were included in the efficacy analysis. The analysis showed that a significant reduction in HAM-A total score for patients treated with duloxetine occurred compared to placebo (10.1 vs 5.9; $P=0.029$ ). Other significant improvements were noted for the HAM-A-psychic factor, HADS anxiety and depression scales. However, significant differences between CGI-I or PGI-I scores compared to placebo, or rates of remission and sustained improvement were not found (24\% vs $7 \%$, $P=0.053$ and $62 \%$ vs $36 \%, P=0.05$, respectively). The study authors concluded the lack of statistical significance was likely due to the relatively small number of study subjects involved in the analysis.

\section{Safety and tolerability}

In general, duloxetine was safe and well tolerated with AEs being mild to moderate in severity. The most common AE was nausea (experienced by about $30 \%$ to $40 \%$ of duloxetine patients) and it was the most frequent reason for study discontinuation due to an AE. Other AEs that occurred more often than placebo were constipation, dry mouth, somnolence, decreased appetite, insomnia, decreased libido, and yawning, although these were regarded as mild and occurred at a relatively low rate. ${ }^{27-31}$ Discontinuation rates due to all AEs for duloxetine patients varied from $2.4 \%$ to $20.2 \% .^{27,28}$

Serious adverse events (SAEs) occurred at a low frequency, and consisted of upper abdominal pain, vomiting, renal cell carcinoma, alcoholism, depression, selfmutilation, ${ }^{29}$ anxiety, atrial fibrillation, back pain, chest pain, migraine, ${ }^{28}$ myocardial infarction, erysipelas, ${ }^{27}$ bronchitis, diarrhea, worsening of GAD, and a ruptured tendon. ${ }^{31}$ There was no significant difference in SAEs between active and placebo groups. There were 2 fatalities (cerebral hemorrhage and suicide), although these were determined to be unrelated to study drug. ${ }^{31}$ Discontinuation emergent adverse events occurred at a rate of $19.9 \%$ to $22.1 \%$, dizziness being the most prominent. ${ }^{27-31}$

In the analysis examining duloxetine in GAD patients with significant pain symptoms, safety and tolerability were not analyzed statistically. For duloxetine use in the elderly population, adverse event rates were similar to those observed among the general population. ${ }^{33}$ Nausea was the most experienced AE, 30\% of duloxetine-treated patients experiencing nausea vs $7.1 \%$ for placebo $(P=0.023)$. In addition, duloxetine-treated patients lost more weight than the placebo group $(-1.1 \mathrm{~kg}$ vs $0.0 \mathrm{~kg}, P=0.018)$. There were no other significant differences between the 2 groups in
AEs or reported lab values. A list of AEs and their frequency is shown in Table 3.

\section{Discussion}

Duloxetine is approved for treatment for GAD in doses of $30 \mathrm{mg}$ to $120 \mathrm{mg}$ daily. Studies have indicated that duloxetine can reduce GAD symptoms. Duloxetine showed significant reductions in HAM-A scores and other measures of GAD. Duloxetine also appears to be relatively well tolerated with AEs being relatively mild and comparable to other those for reuptake inhibitors. Interestingly, in one study, ${ }^{30}$ a $20 \mathrm{mg}$ dose of duloxetine appeared to offer similar efficacy to the higher doses used (60 to $120 \mathrm{mg}$ ). It may be worthwhile conducting a dose-response study to determine if GAD could be successfully treated with duloxetine at doses lower than those currently used, or for clinicians to initiate treatment at $20 \mathrm{mg}$ daily for 4 to 6 weeks before titrating to higher doses. This may help improve patient adherence by reducing the frequency and severity of AEs.

Only one noninferiority comparator study (against venlafaxine XR) has been done, therefore it is difficult to assess whether duloxetine is a more appropriate treatment option other compared with more established and less costly options. Allgulander et $\mathrm{al}^{34}$ analyzed duloxetine vs venlafaxine using data from 2 previous studies. ${ }^{29,30}$ This analysis found a HAM-A score decrease of 3.8 for duloxetine over placebo and of 3.6 for venlafaxine over placebo, which confirmed that duloxetine met criteria for noninferiority against venlafaxine XR. In addition to the efficacy outcome, it was observed that there was no significant difference between the duloxetine and venlafaxine XR groups in the number of patients who discontinued the study because of AEs, or in rate of discontinuation for any specific AE. Both medications had similar tolerability and safety profiles, except for nausea and yawning which occurred at significantly higher rates in duloxetine-treated patients (nausea rates were $26.9 \%$ for duloxetine and $20.1 \%$ for venlafaxine XR $[P<0.05]$, yawning data not reported).

First-line treatment options available for treating GAD involve the use of SSRIs, SNRIs, benzodiazepines, and buspirone. ${ }^{2}$ Benzodiazepines are particularly effective anxiolytics; however, their lack of antidepressant effect and side effect profile make them undesirable for long-term use. Buspirone has good anxiolytic properties, but its negligible antidepressant effect makes it unsuitable for treating the comorbid depression that is highly prevalent in the GAD patient population. Antidepressants such as the SSRI paroxetine, and the SNRI venlafaxine have been shown to be 


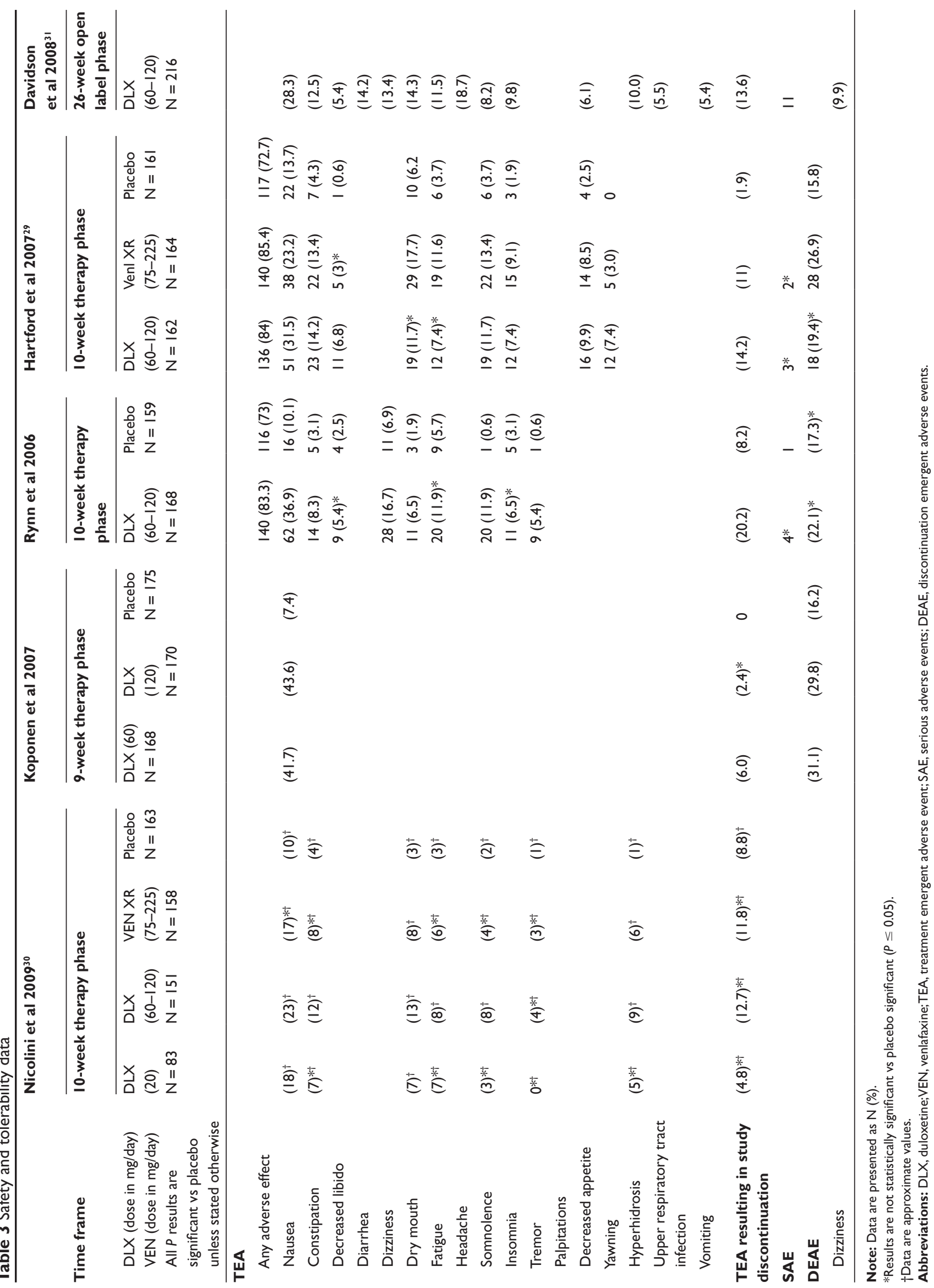


both effective anxiolytics and antidepressants, making them good options for the GAD patient. ${ }^{35}$ Duloxetine, being a SNRI, is one more option at hand from the array of medications available in the SSRI/SNRI class. However, at this time it should be noted there are no direct comparator studies between these different drug classes, thus it is difficult to ascertain the superiority of one treatment over another. Whether clinicians choose to begin treating GAD with an SNRI, SSRI, or other class of medication will ultimately come down to patient history, tolerability to the medication, clinician experiences, and cost. ${ }^{36}$

It should be noted that all five efficacy studies were sponsored by Eli Lilly, who developed and market duloxetine $\left(\right.$ Cymbalta $\left.^{\circledR}\right)$.

\section{Disclosure}

The authors declare no conflicts of interest.

\section{References}

1. American Psychiatric Association. Diagnostic and Statistical Manual of Mental Disorders DSM-IV-TR Fourth Edition. Washington DC: American Psychiatric Association; 1994.

2. Tyrer P, Baldwin D. Generalised anxiety disorder. Lancet. 2006;368: 2156-2166.

3. Grant BF, Hasin DS, Stinson FS, et al. Prevalence, correlates, co-morbidity, and comparative disability of DSM-IV generalized anxiety disorder in the USA: Results from the national epidemiologic survey on alcohol and related conditions. Psychol Med. 2005;35: 1747-1759.

4. Fricchione G. Generalized anxiety disorder. N Engl J Med. 2004;351: 675-682.

5. Wittchen HU. Generalized anxiety disorder: Prevalence, burden, and cost to society. Depression and Anxiety. 2002;16:162-171.

6. Greenberg PE, Sisitsky T, Kessler RC, et al. The economic burden of anxiety disorders in the 1990s. J Clin Psychiatry. 1999;60:427-435.

7. Zhu, Baojin Zhao, Zhongyun Ye, Wenyu Marciniak, Martin D, Swindle R. The cost of comorbid depression and pain for individuals diagnosed with generalized anxiety disorder. J Nervous Ment Dis. 2009; 197:136-139.

8. Hidalgo RB, Davidson JR. Generalized anxiety disorder. An important clinical concern. Med Clin North Am. 2001;85:691-710.

9. Hoffman EJ, Mathew SJ. Anxiety disorders: A comprehensive review of pharmacotherapies. Mt Sinai J Med. 2008;75:248-262.

10. Ballenger JC, Davidson JR, Lecrubier Y, et al. Consensus statement on generalized anxiety disorder from the international consensus group on depression and anxiety. J Clin Psychiatry. 2001;62:53-58.

11. Allgulander C, Bandelow B, Hollander E, et al. WCA recommendations for the long-term treatment of generalized anxiety disorder. CNS Spectrums. 2003;8:53-61.

12. Hunot V, Churchill R, Teixeira V, Silva de Lima M. Psychological Therapies for Generalised Anxiety Disorder. Chichester, UK: John Wiley \& Sons, Ltd; 2007.

13. Cymbalta ${ }^{\circledR}$ [package insert]. Eli Lilly and Company, Indianapolis, IN 46285, USA; 2009.

14. Trivedi MH, Desaiah D, Ossanna MJ, Pritchett YL, Brannan SK, Detke MJ. Clinical evidence for serotonin and norepinephrine reuptake inhibition of duloxetine. Int Clin Psychopharmacol. 2008;23: $161-169$
15. Bymaster FP, Dreshfield-Ahmad LJ, Threlkeld PG, et al. Comparative affinity of duloxetine and venlafaxine for serotonin and norepinephrine transporters in vitro and in vivo, human serotonin receptor subtypes, and other neuronal receptors. Neuropsychopharmacology. 2001;25:871-880.

16. Bymaster FP, Lee TC, Knadler MP, Detke MJ, Iyengar S. The dual transporter inhibitor duloxetine: A review of its preclinical pharmacology, pharmacokinetic profile, and clinical results in depression. Curr Pharm Des. 2005;11:1475-1493.

17. Kasamo K, Blier P, De Montigny C. Blockade of the serotonin and norepinephrine uptake processes by duloxetine: In vitro and in vivo studies in the rat brain. $J$ Pharmacol Exp Ther. 1996;277: $278-286$.

18. Koch S, Hemrick-Luecke SK, Thompson LK, et al. Comparison of effects of dual transporter inhibitors on monoamine transporters and extracellular levels in rats. Neuropharmacology. 2003;45: 935-944.

19. Turcotte JE, Debonnel G, de Montigny C, Hebert C, Blier P. Assessment of the serotonin and norepinephrine reuptake blocking properties of duloxetine in healthy subjects. Neuropsychopharmacology. 2001;24:511-521.

20. Chalon SA, Granier LA, Vandenhende FR, et al. Duloxetine increases serotonin and norepinephrine availability in healthy subjects: A double-blind, controlled study. Neuropsychopharmacology. 2003;28: $1685-1693$.

21. Lantz RJ, Gillespie TA, Rash TJ, et al. Metabolism, excretion, and pharmacokinetics of duloxetine in healthy human subjects. Drug Metab Dispos. 2003;31:1142-1150.

22. Sharma A, Goldberg MJ, Cerimele BJ. Pharmacokinetics and safety of duloxetine, a dual-serotonin and norepinephrine reuptake inhibitor. J Clin Pharmacol. 2000;40:161-167.

23. Skinner MH, Kuan HY, Pan A, et al. Duloxetine is both an inhibitor and a substrate of cytochrome P4502D6 in healthy volunteers. Clin Pharmacol Ther. 2003;73:170-177.

24. Spina E, Santoro V, D'Arrigo C. Clinically relevant pharmacokinetic drug interactions with second-generation antidepressants: An update. Clin Ther. 2008;30:1206-1227.

25. Glueck CJ, Khalil Q, Winiarska M, et al. Interaction of duloxetine and warfarin causing severe elevation of international normalized ratio. JAMA. 2006;295:1517-1518.

26. Strouse TB, Kerrihard TN, Forscher CA, Zakowski P. Serotonin syndrome precipitated by linezolid in a medically ill patient on duloxetine. J Clin Psychopharmacol. 2006;26:681-683.

27. Koponen H, Allgulander C, Erickson J, et al. Efficacy of duloxetine for the treatment of generalized anxiety disorder: Implications for primary care physicians. Prim Care Companion J Clin Psychiatry. 2007;9:100-107.

28. Rynn M, Russell J, Erickson J, et al. Efficacy and safety of duloxetine in the treatment of generalized anxiety disorder: A flexible-dose, progressive-titration, placebo-controlled trial. Depress Anxiety. 2008;25:182-189.

29. Hartford J, Kornstein S, Liebowitz M, et al. Duloxetine as an SNRI treatment for generalized anxiety disorder: Results from a placebo and active-controlled trial. Int Clin Psychopharmacol. 2007;22: 167-174.

30. Nicolini H, Bakish D, Duenas H, et al. Improvement of psychic and somatic symptoms in adult patients with generalized anxiety disorder: Examination from a duloxetine, venlafaxine extended-release and placebo-controlled trial. Psychol Med. 2009;39:267.

31. Davidson JR, Wittchen HU, Llorca PM, et al. Duloxetine treatment for relapse prevention in adults with generalized anxiety disorder: A doubleblind placebo-controlled trial. European Neuropsychopharmacology. 2008;18:673-681.

32. Russell JM, Weisberg R, Fava M, Hartford JT, Erickson JS, D'Souza DN. Efficacy of duloxetine in the treatment of generalized anxiety disorder in patients with clinically significant pain symptoms. Depress Anxiety. 2008;25:E1-E11. 
33. Davidson J, Allgulander C, Pollack MH, et al. Efficacy and tolerability of duloxetine in elderly patients with generalized anxiety disorder: A pooled analysis of four randomized, double-blind, placebocontrolled studies. Human Psychopharmacol. 2008;23:519-526.

34. Allgulander C, Nutt D, Detke M, et al. A non-inferiority comparison of duloxetine and venlafaxine in the treatment of adult patients with generalized anxiety disorder. J Psychopharmacol. 2008;22: $417-425$.
35. Gorman JM. Treating generalized anxiety disorder. J Clin Psychiatry. 2003;64:24-29.

36. Sleath B, Shih YC. Sociological influences on antidepressant prescribing. Soc Sci Med. 2003;56:1335-1344.

\section{Publish your work in this journal}

The International Journal of General Medicine is an international, peer-reviewed open-access journal that focuses on general and internal medicine, pathogenesis, epidemiology, diagnosis, monitoring and treatment protocols. The journal is characterized by the rapid reporting of reviews, original research and clinical studies across all disease areas.
A key focus is the elucidation of disease processes and management protocols resulting in improved outcomes for the patient. The manuscript management system is completely online and includes a very quick and fair peer-review system. Visit http://www.dovepress.com/ testimonials.php to read real quotes from published authors. 